\title{
ADVANCING ALLOY 718 VACUUM ARC REMELTING TECHNOLOGY THROUGH DEVELOPING MODEL-BASED CONTROLS
}

\author{
Rodney L. Williamson ${ }^{1}$, Joseph J. Beaman ${ }^{2}$, Frank J. Zanner ${ }^{3}$ and John J. DeBarbadillo ${ }^{4}$ \\ ${ }^{1}$ Sandia National Laboratories; \\ P.O. Box 5800; Albuquerque, NM 87185-1130, USA \\ ${ }^{2}$ University of Texas; \\ Department of Mechanical Engineering, Austin, TX 78712, USA \\ ${ }^{3}$ Zan Tek Enterprises; \\ P.O. Box 929, Sandia Park, NM 87047, USA \\ ${ }^{4}$ Special Metals Corporation; \\ 3200 Riverside Drive, Huntington, WV 25705, USA
}

Keywords: vacuum arc remelting, model-based controls, VAR process model

\begin{abstract}
The Specialty Metals Processing Consortium (SMPC) was established in 1990 with the goal of advancing the technology of melting and remelting nickel and titanium alloys. In recent years, the SMPC technical program has focused on developing technology to improve control over the final ingot remelting and solidification processes to alleviate conditions that lead to the formation of inclusions and positive and negative segregation. A primary objective is the development of advanced monitoring and control techniques for application to vacuum arc remelting (VAR), with special emphasis on VAR of Alloy 718. This has lead to the development of an accurate, low order electrode melting model for this alloy as well as an advanced process estimator that provides real-time estimates of important process variables such as electrode temperature distribution, instantaneous melt rate, process efficiency, fill ratio, and voltage bias. This, in turn, has enabled the development and industrial application of advanced VAR process monitoring and control systems. The technology is based on the simple idea that the set of variables describing the state of the process must be self-consistent as required by the dynamic process model. The output of the process estimator comprises the statistically optimal estimate of this self-consistent set. Process upsets such as those associated with glows and cracked electrodes are easily identified using estimator based methods.
\end{abstract}

\section{Introduction}

The Specialty Metals Processing Consortium (SMPC) was formed in 1990 and consists of a group of U.S. specialty steel producers and engine manufacturers. One of its primary goals is to eliminate solidification defects in ingots produced with vacuum arc remelting (VAR). To this end, the consortium sponsors research aimed at understanding the formation mechanisms of these defects as well as how better to monitor and control the VAR process. This paper reports recent advances in VAR process monitoring and control resulting from these research activities.

\section{The VAR Process}

Vacuum arc remelting is a process used throughout the specialty metals industry for controlled casting of segregation sensitive and reactive metal alloy ingots. Of particular importance in the former group are nickel-base superalloys such as Alloy 718, commonly used for forged rotating 
parts in the hot stages of jet aircraft engines and increasingly in land-based turbines for power generation. Reactive metal alloys include titanium, zirconium and uranium alloys, a common example being Ti-6Al-4V which is used for a variety of aerospace applications. A number of stainless steels also include VAR as a processing step in their production.

In the VAR process, a cylindrically shaped, alloy electrode is loaded into the water-cooled, copper crucible of a VAR furnace, the furnace is evacuated, and a DC arc is struck between the electrode (cathode) and some start material (e.g. metal chips) at the bottom of the crucible (anode). The arc heats both the start material and the electrode tip, eventually melting both. As the electrode tip is melted away, molten metal drips off and an ingot forms in the copper crucible. Because the crucible diameter is typically $0.05-0.15 \mathrm{~m}$ larger than the electrode diameter, the electrode must be translated downward toward the anode pool to keep the mean distance between the electrode tip and pool surface constant. This mean distance is called the electrode gap $(G)$ and is an important process variable. The objective of VAR is to produce an ingot that is free of macrosegregation, porosity, shrinkage cavities, or any other defects associated with transient solidification during casting. A schematic depiction of the VAR process is presented in Figure 1.

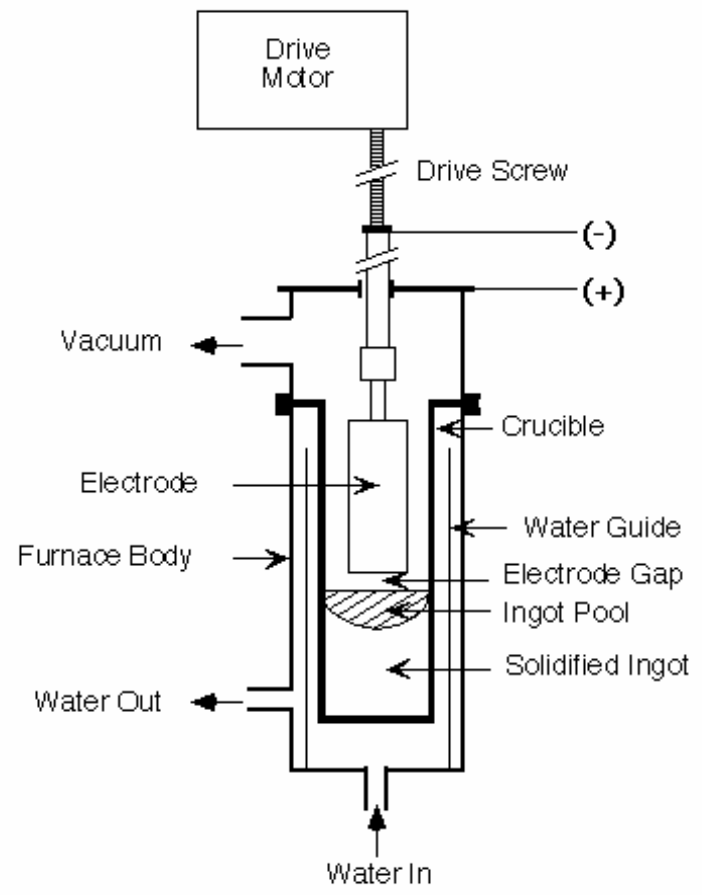

Figure 1. A schematic depiction of a VAR furnace.

\section{Important VAR Process Variables}

In industrial practice, several process variables are monitored and recorded to track the progress and evaluate the status of the VAR process. These include arc voltage $(V)$, melting current $(I)$, electrode position $(X)$ and/or drive velocity $(U=\dot{X})$, drip-short frequency $\left(f_{D S}\right)$ [1], furnace pressure $(P)$, electrode mass $(M)$ and inlet/outlet cooling water temperature $\left(T_{i n}, T_{\text {out }}\right)$. These data streams are usually logged to a computer at regular intervals, displayed on the computer screen, and simultaneously output to a strip-chart recorder. This serves the purpose of generating a permanent record of the process and, at the same time, provides data for evaluating the status of the process for purposes of process monitoring and control.

Electrode melt rate $(\dot{M})$ is an important variable in VAR casting. Melt rate variations cause transients in the ingot growth rate and mushy zone thermal gradient, a condition conducive to the 
formation of melt related defects [2]. For example, such transients have been linked to freckle formation [3] in nickel-base superalloys, as well as solidification white spot formation in Alloy 718 [4]. A method of VAR process control capable of controlling melt rate during transient melting as well as through common melt rate disturbances could lead to significant improvements in product yields as well as reduce the number of melt related defects in segregation sensitive alloys. However, it is very difficult to accurately estimate melt rate using only electrode mass data obtained from load cell transducers. The electrode mass derivative has two properties that lie at the heart of this difficulty: 1) in practice, a derivative is defined on a finite time interval giving rise to an estimate that lags present time and 2) the derivative is an example of a high pass filter and, therefore, melt rate estimates based on electrode weight measurements are very noisy unless some type of averaging technique is applied over a relatively long time period (5-10 minutes). Thus, at best, one can obtain an average melt rate valid at some time in the past. A means of providing accurate, "instantaneous" estimates of melt rate is desired to improve VAR monitoring and control.

Another important variable is process efficiency $(\mu)$ defined as the power that actually goes into electrode melting divided by the total power. Experience has shown that $\mu$ is a sensitive indicator of overall process health, responding to electrode cracks, furnace air leaks, and material cleanliness (e.g. slag contamination from earlier processing steps). Additionally, $\mu$ responds to changes in the electrode temperature distribution such as occur at the very beginning or end of VAR. $\mu$ is not commonly monitored during VAR operation in most shops making it difficult to control melting during normally transient process stages.

Arc voltage can also be a very revealing diagnostic tool in that it reflects furnace atmosphere, arc ionicity, arc distribution and electrode gap. Nominal arc voltage may be modeled with the following equation, which is recognized as a form of Ohm's law:

$$
V=V_{C}+\left(R_{I}+R_{G} G\right) I
$$

$V_{C}$ in this equation is the cathode fall voltage, i.e. the potential difference across the sheath transition region between the electrode surface and the arc plasma column. For Alloy 718, this potential drop is about 21 volts or so and is determined by noting the voltage just prior to arc shorting when the electrode is driven down to touch the ingot pool surface. Thus, for Alloy 718 , almost all the voltage drop between electrode and ingot appears across the cathode sheath. $R_{I}$ and $R_{G}$ are experimentally determined constants. Once characterized, a sustained bias in the measured voltage relative to nominal as predicted by Equation (1) indicates a problem with the arc which may then be further investigated.

Fill ratio is defined as the ratio of the electrode cross-sectional area $\left(A_{e}\right)$ to that of the ingot $\left(A_{i}\right)$. This variable usually changes a little throughout the process because VAR crucibles and electrodes are typically tapered. However, significant voids may exist in cast VAR electrodes due to shrinkage during the casting process. These voids are usually referred to as "pipe." Piped electrodes can cause significant variations in fill ratio, complicating electrode gap and melt rate control. In the equations and models presented below, fill ratio enters in through the variable $a$, defined as $1-A_{e} / A_{i}$.

Two related variables that are crucial to predicting electrode melt rate and gap are the thermal boundary layer $(\Delta)$ and the electrode burn-off rate $(\dot{S}) . \Delta$ is a measure of the distance from the electrode tip at which electrode temperature reaches ambient. $\dot{S}$ is a measure of how fast the electrode length changes due to melting. These dynamic variables are described by the following nonlinear differential equations [5]: 


$$
\begin{aligned}
& \dot{\Delta}=\frac{\alpha_{r} C_{\Delta \Delta}}{\Delta}-\frac{C_{\Delta p} \mu\left[V_{C} I+\left(R_{I}+R_{G} G\right) I^{2}\right]}{h_{m} A_{e}} \\
& \dot{S}=-\frac{\alpha_{r} C_{S \Delta}}{\Delta}+\frac{C_{S p} \mu\left[V_{C} I+\left(R_{I}+R_{G} G\right) I^{2}\right]}{h_{m} A_{e}}
\end{aligned}
$$

where $C_{\Delta \Delta}, C_{\Delta p}, C_{S \Delta}$ and $C_{S p}$ are dimensionless constants derived from material properties. (Values for Alloy 718 are listed in Reference [5].) Melt rate and electrode gap are related to these variables by the following expressions:

$$
\begin{gathered}
\dot{M}=\rho_{s} A_{e} \dot{S} \\
\dot{G}=\dot{S}\left(1-\kappa \frac{A_{e}}{A_{i}}\right)-U=a \dot{S}-U .
\end{gathered}
$$

where $\rho_{s}$ is the density of the liquid metal leaving the electrode at superheat temperature and $\kappa$ is an experimentally measured thermal correction factor to the area ratio.

Because gap can be estimated from drip-short data [6] or arc voltage [7], or a combination of the two [8], using suitable measurement models, it provides an important feedback variable for process control. For Alloy 718, we use the following experimentally determined expression to estimate electrode gap from drip-short data:

$$
G_{D S}=965.0 f_{D S}^{-0.595} I^{-0.669}
$$

Large excursions in electrode gap can have very deleterious effects on ingot properties. As the gap grows large, the electrode tip rounds off and assumes a bullet shape, causing melted electrode material to flow to the center before it drips off into the pool, thus concentrating enthalpy transfer in the center of the ingot. At the same time, as the arc gap becomes larger than the annular dimension between the electrode and crucible wall, the arc partially transfers to the wall. The result is the growth of shelf in from the crucible wall and major perturbation to the pool shape with concomitant changes in the thermal gradient in the mushy zone, the growth rate and the local solidification time. These conditions are conducive to the formation of solidification defects.

Other process variables that have been demonstrated to contain process information are arc plasma temperature as determined by atomic emission spectroscopy [9], magnetic field distribution around the crucible in the water jacket and outside the furnace [10], arc light emission intensity [11], ion distributions in the arc as determined by monochromatic imaging of the arc [12], and temperature, voltage and current distributions in the crucible [13]. Though of importance in specific applications designed to diagnose and investigate the VAR process, these variables are not currently used on a routine basis for practical reasons and are not represented in the process estimator described below.

The VAR Process Estimator

Consider the following set of VAR process variables defining the state vector, $\mathbf{x}$ : 


$$
\mathbf{x}=\left[\begin{array}{c}
\Delta \\
G \\
X \\
M \\
\mu \\
a \\
V_{b}
\end{array}\right] .
$$

$V_{b}$ in this set is the voltage bias variable defined as the average difference between the measured voltage and the nominal voltage defined by Equation (1). Differential equations were given above for all but the last three state variables which are defined as upset or disturbance variables in this formulation. A process disturbance is an event that cannot be controlled through the deterministic inputs of the process, in this case the commanded electrode drive speed $\left(U_{C}\right)$ and current $\left(I_{C}\right)$. A disturbance variable measures a disturbance. For example, unless the pipe in an electrode is exactly characterized ahead of time, the dynamics of $a$ cannot be specified and adjusting the inputs has no effect on the sizes and locations of voids in the electrode. Likewise, there is no way to predict when electrode cracks or contamination will affect process efficiency or voltage. Therefore, the assumption is made that these variables do not change significantly except in response to process upsets, and their associated differential equations are all of the form $\dot{x}_{d}=0$.

Given the normal suite of measurements made during VAR of Alloy 718, namely, $f_{D S}, X_{\text {meas }}$, $M_{\text {meas }}, V_{\text {meas }}$ and $I_{\text {meas }}$, coupled with the differential equations associated with the state variables, it can be shown that all state variables in Equation (9) are uniquely determined in a selfconsistent way, i.e. the system is observable. Thus, even though $\mu, a$ and $V_{b}$ are specified as constants, values for these constants may be estimated and tracked throughout the process. Motions away from their associated nominal values are interpreted as evidence for process upsets. It is assumed that these motions take the form of random walks.

Given the fact that the VAR system state is observable as defined, it is possible to build a Kalman filter based on this system using standard techniques from modern control theory [14]. The outputs of the filter are the optimal state variable estimates in the least squares sense. The filter, then, comprises a VAR process estimator. A full treatment of the mathematical development of the VAR process estimator has been reported elsewhere [15].

\section{Estimator Based Process Monitoring}

The duties of the VAR process monitor are: 1) provide accurate estimates of the state variables; 2) filter the measurement data; 3) detect sensor failures; and 4) detect process upsets. These functions will be briefly discussed below. A block diagram of the monitor is shown in Figure 2.

The VAR Estimator section of the monitor is defined by the Kalman filter described above. Given the commanded inputs from the controller and the suite of filtered measurements provided through the measurements filter, it corrects the model-based estimates of the state vector components and outputs the results. An updated vector is output every $\tau$ seconds. The accuracy of the estimates depends on the accuracy of the dynamic system model, and the precision of the estimates is a direct reflection of the variances used to characterize the noise in the process and 
measurement variables for the system design. If everything has been properly characterized, the estimator provides an accurate, unbiased optimal estimate of the state of the process.

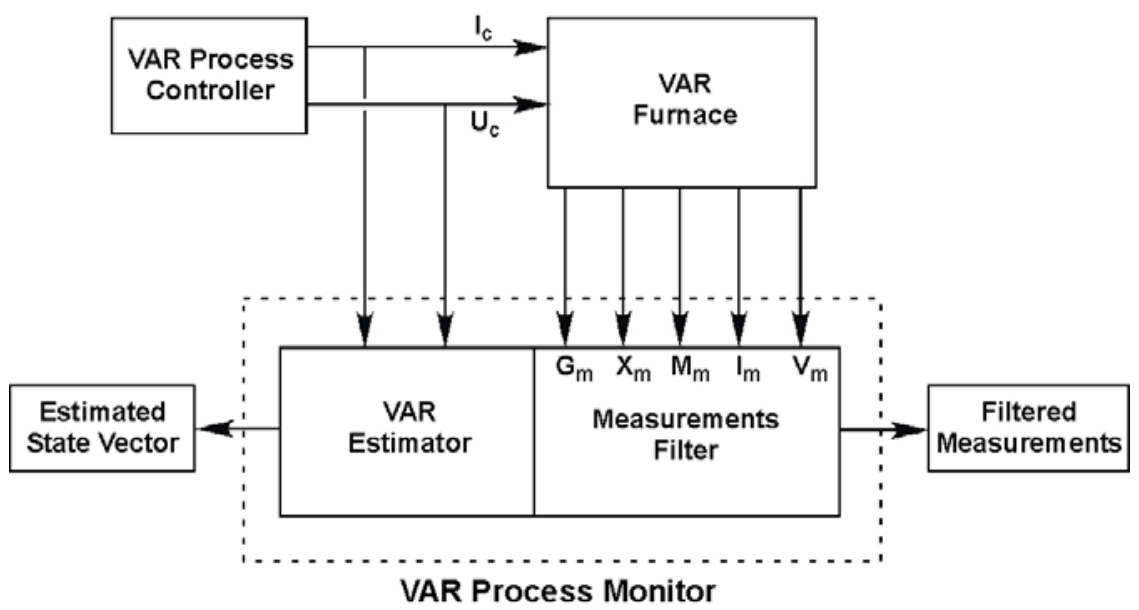

Figure 2. A block diagram of the VAR Process Monitor.

The weaknesses inherent in this estimation scheme are obvious. The estimator is vulnerable to measurement errors and process upsets. A measurement error is any condition that significantly affects either the accuracy or precision of a measurement. Typical causes include broken wires, failed or faulty sensors, electrical interference from nearby machinery, a faulty data acquisition system, and so forth. Irrespective of the error source, if the estimator is fed erroneous measurements, it will provide erroneous estimates. Thus, it is very important that a way be devised to identify faulty measurements and replace them with appropriate estimates. This is what is meant by measurement filtering. However, measurement filtering is complicated by the possible occurrence of process upsets.

Generally speaking, a process upset occurs when the process suddenly or gradually relocates to a region of process space not described by the dynamic model and where the commanded inputs are no longer deterministic. A common example of a VAR upset is known as "glow." Glow can be caused by pressure excursions in the furnace (e.g. due to air leaks or deliberate gas addition) or contamination of the pool surface. During a glow condition, process voltage decreases slightly and becomes nearly independent of electrode gap, melt rate is significantly depressed, and dripshorts either stop or become erratic causing the electrode gap measurement model to yield spurious results. Manipulating current and electrode drive velocity in an effort to end a glow is fruitless. It is generally true of process upsets that one or more data streams become inconsistent with what is expected from the process under normal conditions, often giving rise to a situation where it appears that some measurements have become corrupted or a sensor has failed. The measurements filter must be capable of identifying process upsets so as not to reject good data.

Faulty measurements may arise in very subtle ways and are correspondingly difficult to detect. One example for the VAR process is related to stiction (static friction) between the ram tube seal and the ram tube. In many older furnaces, the furnace is not fitted with a system of load cell transducers that correctly compensates for this effect in the electrode weight measurement, causing apparent fluctuations in melt rate. Stiction events are especially difficult to filter because they happen gradually in a data stream that has already been heavily filtered. There are no sudden electrode weight changes at the beginning of the event to trigger detection, only a gradual apparent change in the first derivative of the electrode weight data. Of course, when the stiction is overcome and the tube breaks free, a sudden change in electrode weight is observed, but the event is over at this point. The effects of stiction events on the process are of minimal impact if the load cell output is not being used as feedback to actively control melt rate. But if it is being 
used in such a control scheme, true melt rate fluctuations will result as the controller reacts to the corrupted weight measurements. If the data are filtered using conventional methods, e.g. least squares averaging, controller responsiveness will suffer. Ideally, one would like to detect the events near their onsets and correct the data as they are collected so that the events become "transparent."

To summarize, the measurements filter must accomplish the following tasks: 1) distinguish between measurement errors and process upsets; 2) identify transient measurement errors and supply corrected data; 3 ) identify and flag measurement failures.

The measurements filter utilized in the VAR process monitor developed by the SMPC relies on a technique generally known as residual monitoring [16]. The residuals of interest are those comprising the innovations vector. Each element in this vector is the difference between a process variable's measured value and its model-based estimate. If the residuals are tracked over time, they should average to zero if the dynamic system model is unbiased, and their respective variances should remain relatively constant. These two criteria form the basis of the filter. When the filter detects a faulty data point, it rejects it and replaces it with data from the estimator. When it detects a process upset, it retains the data and flags the event. A complete description of this filter is well beyond the scope of this paper. However, Figure 3 shows an example of faulty electrode weight data that have been rejected and replaced with the estimated electrode weight data based on the filtered measurements. The arrows in the figure point to plateaus in the data related to ram tube seal stiction events. These data were obtained during VAR of $0.43 \mathrm{~m}$ diameter Alloy 718 electrode into $0.51 \mathrm{~m}$ diameter ingot.

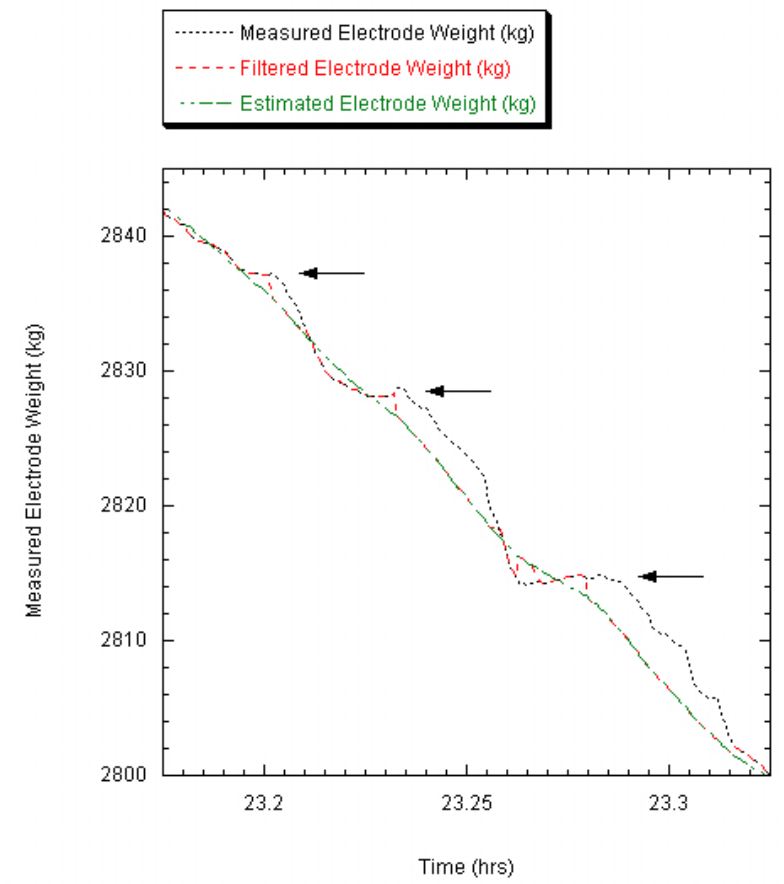

Figure 3. An example of ram tube seal stiction events (arrows) in load cell data that has been filtered using a model based measurements filter.

\section{Estimator-Based Control of the VAR Process}

The output from the estimator-based VAR monitor may be used to build a feedback controller for the VAR process. Given that the process has two deterministic inputs, we may choose to control two process variables. In this instance, we choose to control electrode gap and melt rate. Figure 4 shows a schematic diagram of the controller. $f_{P}, f_{i}$ and $f_{G}$ in this diagram are nonlinear functions obtained by inverting the dynamic model [17]. 


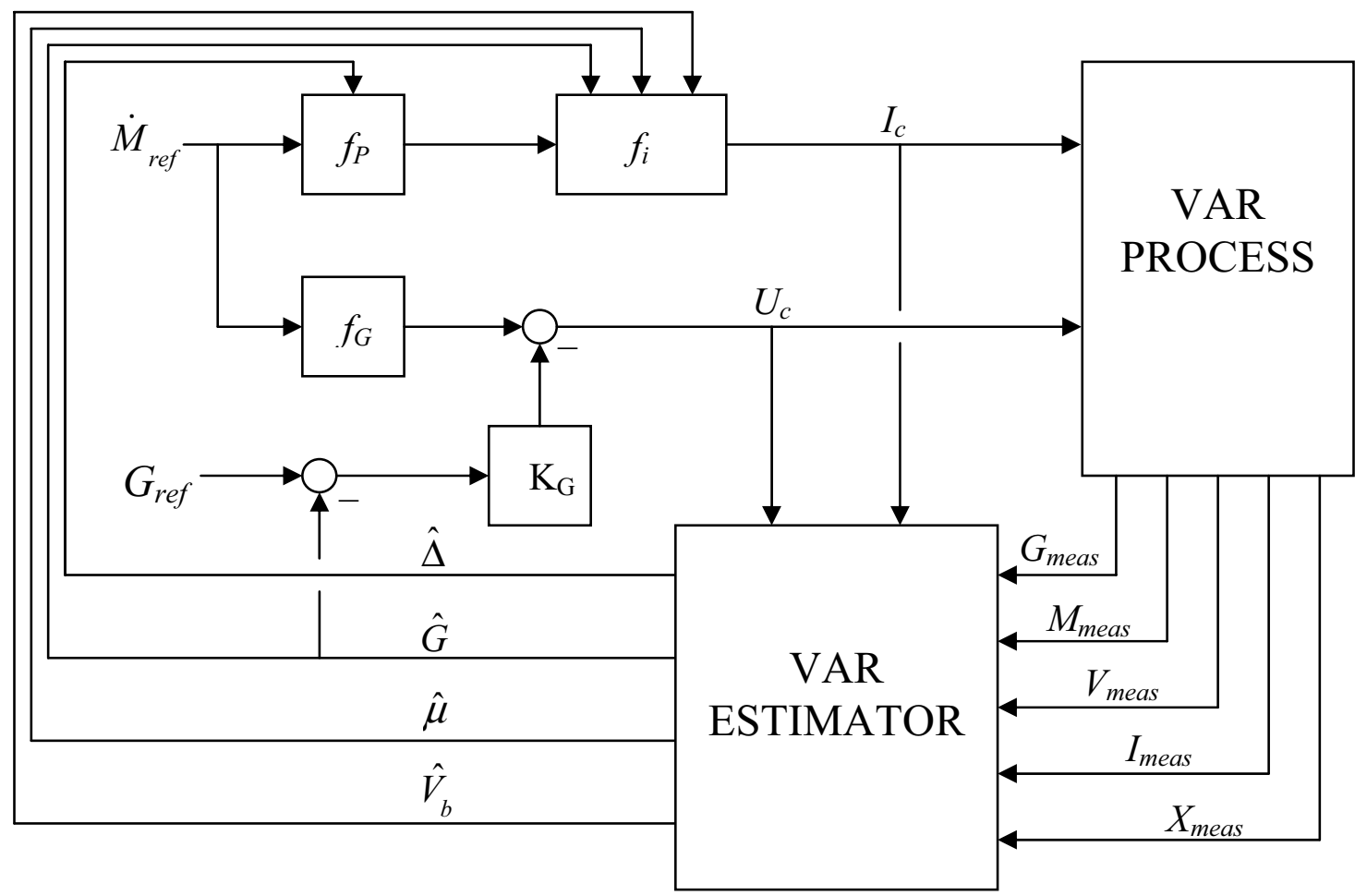

Figure 4. Schematic diagram showing a VAR process estimator based melt rate and gap controller.

An example of how well this type of model-based control works is shown in Figure 5. This figure shows a plot of electrode weight data acquired during VAR of a $0.43 \mathrm{~m}$ diameter Alloy 718 electrode into $0.51 \mathrm{~m}$ diameter ingot. The electrode had been cut approximately half way through its diameter with an abrasive saw to provide a significant perturbation to thermal conduction in the electrode. Also plotted in the figure are the melt rate reference setpoint and the average melt rate obtained by doing a 5 minute running least squares analysis of the weight data and finding the slope at each point along the weight curve. A perturbation to the average melt rate is observed between 14.5 and 14.7 hours. Using standard methods of melt rate control, this perturbation to the average melt rate would have been much larger, typically around $\pm 50 \%$. This technology is very useful when trying to control melt rate during startup or hottop where the thermal distribution in the electrode has not reached or has been driven away from steady state.

\section{Conclusions}

Low order process models have been developed and assembled into a model of the VAR process. The process model has been used to develop a sophisticated process monitor, measurements filter, and process controller. This technology provides means to distinguish between measurement errors and process upsets. It also provides a way of correcting faulty measurements using model-based estimates. A dynamic melt rate controller has been demonstrated that can control average melt rate through large process upsets caused by variations in heat conduction in the electrode.

\section{Acknowledgement}

A portion of this work was supported by the United States Department of Energy under Contract DE-AC04-94AL85000. Sandia is a multiprogram laboratory operated by Sandia Corporation, a Lockheed Martin Company, for the United States Department of Energy. Support was also 
provided by the U.S. Federal Aviation Administration and the Specialty Metals Processing Consortium.

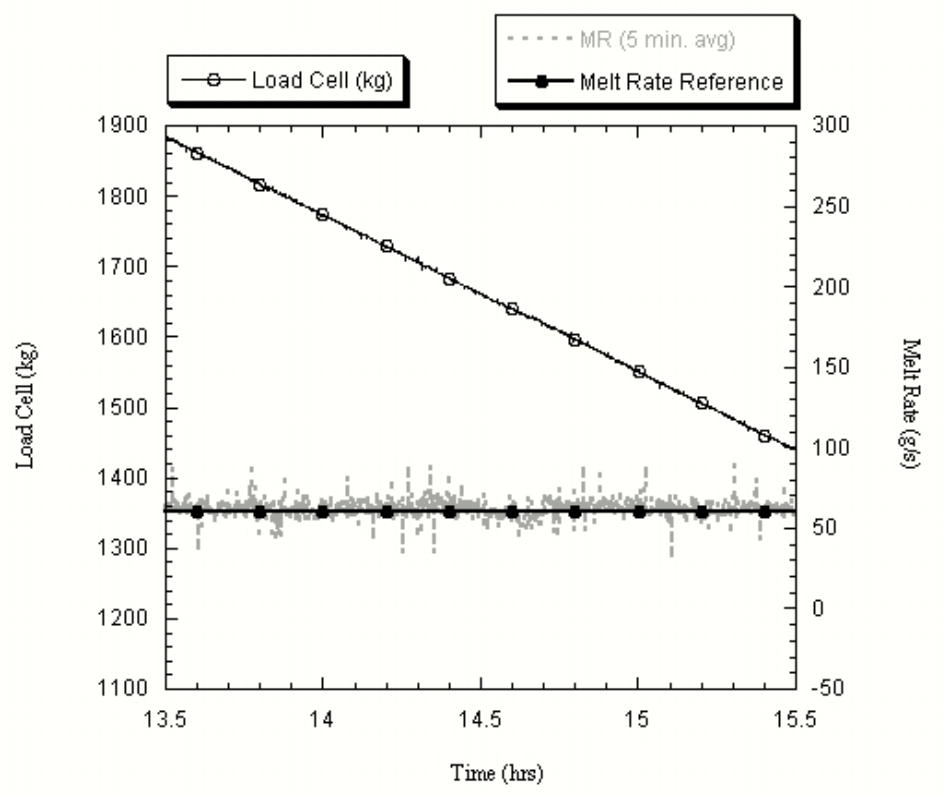

Figure 5. Plot of electrode weight data and average (5 minute) melt rate while melting through an electrode that had been cut half way through its diameter with an abrasive wheel.

\section{References}

1. Drip-shorts are momentary arc interruptions caused by metal drips bridging the electrode gap and contacting the ingot pool surface. See F. J. Zanner, Metall. Trans. B, 10B (1979), 133-42 for a detailed description of drip-short signatures.

2. M.C. Flemings, Solidification Processing (New York, NY: McGraw-Hill, 1974), 245.

3. T. Suzuki et al., Proc. of The 2001 Int'l Symposium on Liquid Metal Processing And Casting, ed. A. Mitchell and J. Van Den Avyle (Santa Fe, NM: American Vacuum Society, 2001), 32537.

4. L.A Bertram et al., Proc. of The 1999 Int'l Symposium on Liquid Metal Processing And Casting, ed. A. Mitchell, L. Ridgway and M. Baldwin (Santa Fe, NM: American Vacuum Society, 1999), 156-67.

5. R.L. Williamson, J.J. Beaman, D.K. Melgaard: Met. and Mat. Trans. B, 35B (2004), 101-13.

6. F.J. Zanner: Met. Trans. B, 12B (1981), 721-28.

7. R.L. Williamson, F.J. Zanner, and S.M. Grose: Met. and Mat. Trans. B, 28B (1997), 841-53.

8. C.L. Hysinger et al., Proc. of The 1999 Int'l Symposium on Liquid Metal Processing And Casting, ed. A. Mitchell, L. Ridgway and M. Baldwin (Santa Fe, NM: American Vacuum Society, 1999), 145-55. 
9. R.L. Williamson and S.M. Grose, Proc. of the 1994 International Symposium on Liquid Metal Processing and Casting, ed. A. Mitchell and J. Fernihough (Santa Fe, NM: American Vacuum Society), 130-42.

10. R.M. Ward and M.H. Jacobs, Proc. of The 2001 Int'l Symposium on Liquid Metal Processing And Casting, ed. A. Mitchell and J. Van Den Avyle (Santa Fe, NM: American Vacuum Society, 2001), 244-57.

11. R.L. Williamson, unpublished results.

12. R.L. Williamson and M.E. Schlienger, Proc. of the XVIIth International Symposium on Discharges and Electrical Insulation in Vacuum, Vol. 1, ed. A. Anders and S. Anders (Berkeley, CA:, 1996), 113-17.

13. R. L. Williamson, G. J. Shelmidine, Proc. of 5th International Special Emphasis Symposium of Superalloys 718, 625, 706 and Derivatives, ed. E. A. Loria (Pittsburgh, PA: TMS, 2001), 91102. Also, see Reference [10].

14. See any text on control theory and application, e.g. B. Friedland, Control System Design: An Introduction to State-Space Methods (New York, NY: McGraw-Hill, 1986).

15. J. J. Beaman, R. L. Williamson and D. K. Melgaard, Proc. of The 2001 Int'l Symposium on Liquid Metal Processing And Casting, ed. A. Mitchell and J. Van Den Avyle (Santa Fe, NM: American Vacuum Society, 2001), 161-74.

16. P. S. Maybeck, Stochastic Models, Estimation, and Control, Vol 1 (New York, NY: Academic Press, 1979), 229-31.

17. J.J. Beaman et al., J. Material Science 39 (2004), 7153-59. 